\section{Taxonomists and stability}

SIR-The erroneous assumption has been made in recent critiques of the frequent name-changing by certain taxonomists that only two camps are involved: the "taxonomists, who want to change the names of organisms, and other biologists who do not" (J. A. Barnett, Nature 338, $547 ; 1989)$. Others in earlier communications have made similar statements Actually, there is a large minority (perhaps actually a majority) of taxonomists who are as anxious to retain the stability of names as the average biologist. These biologically orientated taxonomists fully realize that names are the keys to a vast information storage and retrieval system and that every change of a name corresponds to a change of a key, resulting in confusion and loss of information.

In discussing stability, one must clearly distinguish between inevitable changes in a name, such as when an incorrectly classified species is transferred to the genus to which it belongs, and changes caused only by a rigid application of certain arbitrary rules of nomenclature. To minimize the number of the latter changes, zoologists had for years a set of rules automatically protecting universally used names.

These rules were unfortunately rejected by the majority of delegates attending a nomenclature meeting at the International Congress of Zoology in 1963. Many taxonomists have since pleaded for the restoration of these stabilizing provisions. Restoring them would go a long way towards stabilizing zoological nomenclature, a goal surely supported by the majority not only of biologists but even of taxonomists. It would also greatly reduce the workload of the International Commission on Zoological Nomenclature and its secretariat.

Museum of Comparative Zoology,

Harvard University,

Cambridge, Massachusetts 02138, USA

\section{The grants game}

SIR-A form of lying has become an accepted commonplace in science. Its systematic use may help to explain the recent rash of reports of scientific fraud.

The 'lies' are grant proposals. Kendrew has pointed out that in order to obtain a research grant, a scientist "has to cheat. If he expressed his own motivation honestly he would get no money"'. Angier quotes Weinberg as another who believes that to be the case ${ }^{2}$.

Kendrew and Weinberg are right. All innovative scientists know that they would rarely be funded, such is the nature of the review system, if they truthfully set out what they actually intended to do and what their motivation was for doing it. So we all tell acceptable lies.

What do we do when faced with the necessity of telling a lie in order to achieve what we believe to be legitimate ends? All of us believe that we know the difference between real lying, which is unforgivable and subverts science, and acceptable lying which we must do so that we can obtain the funds to discover the truth. We believe that we can repeatedly lie in our grant proposals and yet know when to stop when we write up the results.

But do we? Do even the best among us, once we step over the line which says that truth and honesty are indivisible, know any more what is truly right and truly wrong? And what about those who are less than the best, who are less clearsighted, whose self-confident morality is less robust? What does the blurring of values, the elision of acceptable and unacceptable practices, the confusion of different classes of truth, do to them? Might it not be rather difficult for them to distinguish between the acceptable lies we must tell in a grant proposal and the unacceptable ones we might tell when writing our results or when reviewing a competitor's grant application or paper?

If we cannot do better than collude in a system which requires us to tell lies in order to obtain money to seek the truth, we deserve neither the public's trust nor its funding.

Efamol Research Institute, DAVID F. HORROBIN

Annapolis Valley Industrial Park,

Kentville, Nova Scotia,

Canada $B 4 N 4 H 8$

1. Kendrew, J. in Priorities in Research (eds Kendrew, J. \& Shelley, J.) p. 171 (Excerpta Medica, Amsterdam, 1983)

Angier, N. in Natural Obsessions: The Search for the Oncogene. p. 24 (Houghton Mifflin, Boston, 1988).

\section{On principles}

SIR-John Barrow (Nature 339, 170; 1989) calls into question our report (Nature 337, 411-412; 1989) on the recent conference in Venice on the anthropic principle in cosmology. We find it difficult to see that Barrow's letter accurately conveys the main thrust of his contribution to the conference.

Anyone seriously interested in this topic will know that there is a range of principles proposed, from the weak anthropic principle (WAP), through the strong anthropic principle (SAP), to the final anthropic principle (FAP) put forward by Barrow and Tipler in their major book ${ }^{i}$. The first is unexceptionable and the second intriguing but highly debatable, while the last is so difficult to accept that its advocacy tends to bring the whole topic into disrepute (see for example Martin Gardner's comments in the New York Review ${ }^{2}$ ).

At the Venice conference, Barrow gave a wide-ranging and thought provoking talk on "Patterns of explanation in cosmology", in which the weak anthropic principle was put forward as having a powerful explanatory role. He also commented on the strong anthropic principle, but did not even mention the final anthropic principle. Just as in Sherlock Holmes' comment on the strange incident of the Dog in the Night (what did he do? nothing!), we believe the listener to that talk was entitled to conclude Barrow did not at that time regard the FAP as a significant principle of explanatory power in cosmology (else surely it would have been mentioned). Thus our comment that he had abandoned the FAP.

His letter to Nature states that he defended the FAP in later discussions arising from other people's talks. Thus, to be technically correct, we should have merely said that he did not mention the issue in his major review. We believe the implication remains (and is of interest to your readers): a serious review of explanation in cosmology will not include the FAP. This was certainly the impression conveyed to us, and others, by Barrow's presentation.

MAREK ABRAMOWICZ GEORGE ELLIS

SISSA,

Strada Costiera 11,

Miramare, Trieste 34014, Italy 1. Barrow, J.D. \& Tipler, F.J. The Anthropic Cosmological
Principle (Oxford University Press, 1986).

2. Gardener, M. The New York Review 22-26 (8 May 1986)

\section{Anonymous review}

SIR-Alun Anderson writes: "Eliminating bias by hiding the names and affiliations of authors and the identity of reviewers had little effect on editors' recommendations" (Nature 339, 164; 1989). Most editors have such a wide range of material to deal with that they are not normally the best people to recognize bias. Authors are.

The identity or affiliation of authors has never been hidden from this reviewer, whereas reviewers' identities are nearly always hidden from authors - at least in my experience and that of people with whom I have discussed the matter.

Bias would be more explicitly revealed if reviewers signed their names, even though it could not be eliminated. Reviewers would also write more carefully considered reports. I believe that would go some way towards eliminating errors. Then, even if papers did "make their way down the hierarchy of journals", they would at least by corrected, modified and improved en route.

Department of Earth Sciences,

Open University,

Walton Hall.

Milton Keynes MK7 6AA, UK 